\title{
Do low-confidence individuals decrease group judgments' accuracy? Investigations in terms of the wisdom of crowds framework
}

Masaru Shirasuna ( $\sim$ m.shirasuna1392@gmail.com )

Otemon Gakuin University

Hidehito Honda

Otemon Gakuin University

\section{Research Article}

Keywords: Confidence, Wisdom of crowds, Individual judgments, Group judgments, Computer simulations

Posted Date: September 14th, 2021

DOI: https://doi.org/10.21203/rs.3.rs-900388/v1

License: (c) (i) This work is licensed under a Creative Commons Attribution 4.0 International License.

Read Full License 


\section{Abstract}

In group judgments in a binary choice task, the judgments of individuals with low confidence (i.e., they feel that the judgment was not correct) may be regarded as unreliable. Previous studies have shown that aggregating individuals' diverse judgments can lead to high accuracy in group judgments, a phenomenon known as the wisdom of crowds. Therefore, if low-confidence individuals make diverse judgments between individuals and the mean of accuracy of their judgments is above the chance level (.50), it is likely that they will not always decrease the accuracy of group judgments. To investigate this issue, the present study conducted behavioral experiments using binary choice inferential tasks, and computer simulations of group judgments by manipulating group sizes and individuals' confidence levels. Results revealed that (I) judgment patterns were highly similar between individuals regardless of their confidence levels; (II) the low-confidence group could make judgments as accurate as the high-confidence group, as the group size increased; and (III) even if there were low-confidence individuals in a group, they generally did not inhibit group judgment accuracy. The results suggest the usefulness of low-confidence individuals' judgments in a group and provide practical implications for real-world group judgments.

\section{Introduction}

\subsection{Wisdom of crowds and individuals' confidence about their own judgments}

Individuals with high confidence (often experts) sometimes have much knowledge about tasks. Especially in group-judgment situations, their judgments may seem to be more accurate and reliable, and contribute to improving the accuracy of group judgments. Thus, the judgments of high-confidence individuals may be more likely to be accepted by group members. In contrast, some individuals with low confidence (often novices) may have less knowledge and have low confidence regarding tasks. Judgments made by low-confidence individuals may seem to be less accurate and unreliable, and therefore may impair the accuracy of group judgments.

However, can it be considered truly worse for a group to have low-confidence individuals? The present study defined group judgments as judgments made by group members using a majority rule (i.e., the group chooses one alternative that was most chosen by group members in a binary choice task). Then, the study focused on the relationships between individuals' subjective confidence about their own judgments and the accuracy of group judgments in a binary choice task. Generally, low-confidence individuals may have less knowledge about tasks than high-confidence individuals and may sometimes have to guess the answer. Therefore, at the individual level, these individuals may tend to make less accurate judgments compared to high-confidence individuals. However, at the group level, it is expected that such a lack of knowledge by low-confidence individuals may not always impair the accuracy of group judgments, based on the framework of the wisdom of crowds. Previous studies have shown that simply aggregating individuals' diverse judgments (e.g., by a majority rule) can often lead to highly 
accurate judgments in group levels ${ }^{[1-3]}$, which is known as the wisdom of crowds ${ }^{[2-10]}$. To achieve the wisdom of crowds by using a majority rule in a binary choice task, one of the important aspects is that the mean of individuals' rates of correct judgments is above the chance level, . $50^{[2][10]}$. It is also important that individuals do not make the same judgments as one particular person and they show diverse patterns of judgments ${ }^{[2],[10-11]}$. When low-confidence individuals make judgments based on their lesser knowledge that involves high uncertainty, their judgments tend to be diverse among individuals. In a binary choice task, as long as their accuracy is above .50 at the individual level, the accuracy of judgments at the group level can be boosted by aggregating individuals' judgments using a majority rule (conceptually, see Fig. 1). By focusing on the wisdom of crowds in terms of individuals' confidence, it is expected that we may be able to provide further understanding and practical implications regarding group judgments.

\subsection{Study outline}

To obtain the first evidence about the relationships between individuals' subjective confidence and group judgments' accuracy, the present study conducted behavioral experiments and computer simulations. To investigate the validity of the results, two types of inferential tasks were used: a population inference task $^{[12-16]}$ and a relationships comparison task ${ }^{[17-19]}$ (both of which are described later in detail). First, online behavioral experiments were conducted wherein individuals were asked to answer binary choice questions and rate subjective confidence about their judgments. Then, computer simulations of group judgments were performed using the obtained behavioral data, to evaluate the performances of group judgments. We manipulated the group sizes (i.e., the number of members in a group) and the proportions of individuals' confidence levels (i.e., low, medium, and high) in a group, and then evaluated the accuracy of group judgments by applying a majority rule.

\section{Method}

In the behavioral experiments, two types of tasks were used, as described earlier (i.e., a population inference task and a relationships comparison task), using the same procedure. The protocols of the cognitive experiments in the present study conformed to the Declaration of Helsinki and were approved by the Ethics Review Committee for Experimental Research at Otemon Gakuin University.

\subsection{Participants}

Of a total of 601 participants, 302 people were assigned to a population inference task $\left(n_{\text {male }}=179\right.$, $\left.n_{\text {female }}=120, n_{\text {other }}=3 ; M_{\text {age }}=41.2, S D_{\text {age }}=9.52\right)$, and 299 were assigned to a relationships comparison task $\left(n_{\text {male }}=167, n_{\text {female }}=130, n_{\text {other }}=2 ; M_{\text {age }}=42.8, S D_{\text {age }}=10.1\right)$. To verify the robustness and replicability of our experimental results, each task was split into two sets (i.e., half the population inference questions as "population inference set A" and the other half as "population inference set B"; further, half the relationships comparison questions as "relationships comparison set $A$ " and the other 
half as "relationships comparison set B"), and participants were randomly assigned to one of these task sets (in a population inference task, $n_{\text {setA }}=152, n_{\text {setB }}=150$; in a relationships comparison task, $n_{\text {setA }}=$ $\left.151 ; n_{\text {setB }}=148\right)$. Since highly consistent results were obtained between sets $A$ and $B$, hereafter in the main text, the results of one of two sets for each task (i.e., "population inference set A" and "relationships comparison set $A^{\prime \prime}$ ) will reported for simplification. The sample sizes were set as follows: Because this study was the first to investigate the relationships between subjective confidence and accuracy of group judgments, medium effect sizes were anticipated in the one-way analysis of variance (ANOVA) (as described later, we focused on differences of group judgments' accuracy between three confidence levels: low, medium, and high); if the effect size was 0.25 , then the required sample size was 159 , while if it was 0.30 , then the required sample size was 111 . Therefore, the sample sizes were set to approximately 150 for each task set. These sample sizes were calculated using GPower $3.1^{[20]}$.

Participants were recruited via a Japanese crowdsourcing service, Lancers (https://www.lancers.jp/). Participants were rewarded 500 JPY for their responses upon completion of the experiment.

\subsection{Tasks and materials}

Population inference task (Fig. 2, upper): Many previous studies have used a population inference task to examine human inferences such as heuristics ${ }^{[21-23]}$, In this task, typically, the task structure is that two objects (city names) are presented as alternatives, for example "Which city has a larger population, city A or city B?" Participants are asked to simply compare these two alternatives and make inferences in terms of which city has a larger population. Regarding the objects presented to participants in the current study, we used parts of city names that were used in a previous study ${ }^{[13]}$. For the detailed procedure concerning the selection of questions and the question list, see Supplementary Information 1.

Relationships comparison task (Fig. 2, lower): A relationships comparison task is a task recently proposed for examining human inferences (especially, the adaptive use of heuristics within the adaptive toolbox framework) ${ }^{[18],[19]}$. In this task, the task structure is that objects are presented not only in alternatives but also in a question sentence format, for example "Which country is city $Q$ in, country $A$ or country B?" In the present study, the relationships comparison task was used as another task to investigate the effects of individuals' confidence in their judgments on group judgments. Regarding the objects that were presented to participants, we selected 50 cities and 21 countries used in Study $2 \mathrm{~b}$ of the

previous study ${ }^{[18]}$ based on the correct rate of each question in the original study. For details on the process of selecting questions and the question list, see Supplementary Information 2.

\subsection{Procedures}

All tasks were conducted online, and the graphical user interface (GUI) was performed using Qualtrics (https://www.qualtrics.com/jp/). The general procedure in the behavioral experiments was identical between the two types of tasks. Participants were asked to make an inference for a population inference task (e.g., "Which city has a larger population, Yokohama or Chiba?") or a relationships comparison task (e.g., Which country is Addis Ababa in, Ethiopia or Egypt?), and then to rate their subjective confidence 
about the judgment (i.e., "How confident do you feel for your judgment?") using a visual analog scale in which the left and right ends indicate 0 ("not confident at all") and 100 ("highly confident"), respectively. After choosing one of the two alternatives and rating their confidence, the next question was presented on a computer screen. The order of presenting questions and presenting the two alternatives were all randomized. To check whether participants faithfully answered the tasks online, we inserted several questions, called "check problems" (e.g., "Choose 'Japan' in this question. Japan or Tokyo"). Participants who did not correctly respond to the check problem (e.g., chose "Tokyo" in the above example) were excluded from the analyses. The differences between a population inference task and a relationships comparison task were with respect to the number of questions and the positioning of check problems. In a population inference task, 70 questions were presented for each participant and the check problem was inserted after the 35th question; while in a relationships comparison task, 25 questions were presented and the check problem was inserted after the 13th question.

\subsection{Data exclusion and confidence categorization}

Participants were excluded from the analyses based on the following two criteria: participants whose total response time was less than five min or above $50 \mathrm{~min}$, and participants who did not answer the check problems as instructed. Consequently, this study used data from 296 participants ( $n_{\text {setA }}=150 ; n_{\text {setB }}$ $=146)$ in the population inference task, and 296 participants $\left(n_{\text {setA }}=149 ; n_{\text {setB }}=147\right)$ in the relationships comparison task.

Next, for each question, participants were categorized into three confidence levels based on their ratings of subjective confidence: low, medium, or high.

\subsection{Computer simulations of group judgments}

Using data from behavioral experiments, computer simulations of group judgments were conducted to investigate the relationships between individuals' confidence and judgment accuracy. Specifically, the group sizes were manipulated in five patterns: 1 (i.e., situation in individual judgments), 5, 10, 20, and 30 (i.e., situations in group judgments). Subsequently, simulations were conducted in the following manner:

- For group size 1, the mean rates of correct judgments (i.e., accuracy) were calculated for each question.

- For group sizes 5, 10, 20, and 30, we randomly generated 5,000 groups and assumed that group judgments were made by a simple majority rule. Note that if the number of agents who chose one alternative was equal to that of agents who chose the other alternative, then a group chose one of two alternatives randomly. The mean accuracy in the 5,000 groups was then calculated for each question.

When generating groups, we set two types of groups. First, this study set groups in which individuals' confidence levels were similar to each other (called "similar confidence" groups). For each question, 5,000 groups were generated by collecting individuals from one confidence level (i.e., low, medium, or high) (Fig. 3, upper). 
Second, this study set groups which included individuals with diverse confidence levels (called "diverse confidence" groups). For each question, 5,000 groups were generated by collecting individuals from several confidence levels. When collecting individuals, we manipulated the proportions of confidence levels in each group. Specifically, this study set the following 10 proportions: (Low: Medium: High) $=(3: 1$ : 1), (3: 2: 0), (3:0:2), (1:3:1), (2: 3: 0), (0:3:2), (1:1:3), (2:0:3), (0:2:3), and (1:1:1) (Fig. 3, lower). Note that, although all 10 patterns for each group size were analyzed, this paper reports the results only in the proportion (1:1:1) in the main text and show the other results in Supplementary Information 6 , because the results showed similar tendencies in all proportions and group sizes.

\section{Results}

As previously described, the main text reports set A's results for each task (i.e., "population inference set $A$ " and "relationships comparison set A"), as the results of sets $A$ were highly consistent with those of set $B$ (i.e., "population inference set B" and "relationships comparison set B"). Set B's main results obtained from the computer simulations are shown in Supplementary Information 3. All data, code, and materials used in the experiments are available at https://osf.io/ehzu6/? view_only=fb08ab0fc87c45eabb201316bd2c0881.

\subsection{General tendencies of relationships between confidence and accuracy in individual levels}

First, this study confirmed the general tendencies of relationships between individuals' confidence ratings and their accuracy of judgments at individual levels. Superficially, it seemed that when individuals had higher confidence in their judgments, they would be able to make more accurate judgments, and vice versa. It was speculated, however, that such tendencies might not always hold true: even if individuals had higher confidence, they might sometimes make less accurate judgments (e.g., overconfidence ${ }^{[24-26]}$ ).

This study investigated the relationships between confidence and accuracy for each question at individual levels (i.e., group size 1) by using a generalized linear model, and the typical results are shown in Fig. 4. As evident in these graphs, higher-confidence individuals do not always make more accurate judgments. To confirm such tendencies in more detail, we calculated the coefficients of confidence in the generalized linear models for each question, and the distributions of the coefficients are shown in Fig. 5. As shown in these histograms, both positive and negative coefficients are observed. That is, even if individuals had higher confidence, they made inaccurate judgments as well as accurate judgments with higher confidence. In short, individuals' subjective confidence and accuracy of judgments had generally positive correlations, but were not highly correlated to each other. In Supplementary Information 4 and 5, we showed tendencies in all questions, and also estimated coefficients of individuals' confidence on the accuracy of their judgments using a generalized linear mixed model (i.e., parameter estimations of models including random effects of questions and participants) in order to rigorously investigate the relations between confidence and accuracy. 
Furthermore, we confirmed differences in judgment patterns between the confidence levels for each question. For each confidence level (i.e., low, medium, and high), we calculated the rates of correct judgments for each question at individual levels, and then plotted the relationships between these rates. In Fig. 6, each point denotes the rate of correct judgments for each question. As shown in Fig. 6, similar judgment patterns are observed among all confidence levels because several points lay on the first or third quadrant. The proportions of points in the first or third quadrant were .88 (low-medium), .87 (lowhigh), and .91 (medium-high) in a population inference task; and .64 (low-medium), .68 (low-high), and .72 (medium-high) in a relationships comparison task. These results suggest that low-confidence individuals will not always decrease the accuracy of group judgments because their judgment patterns are similar to medium- and high-confidence individuals' judgment patterns. The next section investigates this suggestion through computer simulations of group judgments.

\subsection{Computer simulations of group judgments in similar and diverse confidence groups}

Computer simulations were conducted to investigate the performances of judgments in the two types of groups: "Similar confidence" groups (i.e., confidence levels in a group were similar between individuals), and "diverse confidence" groups (i.e., confidence levels in a group differed between individuals).

According to the framework of the wisdom of crowds, the accuracy of group judgments in a binary choice task can improve when the mean of rates of individuals' correct judgments is above the chance level, .50 . Even if individuals' confidence was lower, their judgments did not always decrease group judgment accuracy when their accuracy was above .50 at individual levels. The present study investigated this issue using the first computer simulation for similar confidence groups.

The results of a population inference task and those of a relationships comparison task are shown in Fig. 7. In both tasks and at all confidence levels (i.e., low, medium, and high), the accuracy was above the chance level (.50) in group size 1, and the accuracy of group judgments did not fall below .50, regardless of individuals' confidence levels. Importantly, as observed in a population inference task, even if lowconfidence individuals made significantly less accurate judgments than high-confidence individuals (low .67 and high .75 in group size 1), low-confidence groups could make judgments as accurate as highconfidence groups (low .78 and high .80 in group size 30 ) because low-confidence individuals' accuracy was significantly higher than .50 . In a relationships comparison task, on the other hand, the accuracy in low-confidence individuals was not so high (.54 in group size 1), and that in low-confidence groups did not get better or worse substantially (.54 in group size 30 ). These results suggest that, even if lowconfidence individuals exist in a group, they will not always inhibit group judgment accuracy as long as the mean of individuals' accuracy is above the chance level.

As stated previously, this study also examined the situations where individuals' confidence levels differed between each other in a group? Individuals with high confidence may generally be people who have much knowledge about tasks, while individuals with low confidence may have little knowledge. Based on the framework of the wisdom of crowds and on the results of the first computer simulation, however, the 
accuracy of group judgments may not be inhibited even when the group considers low-confidence individuals' judgments. The present study investigated this issue using the second computer simulation involving diverse confidence groups. The results of a population inference task and those of a relationships comparison task are shown in Fig. 8, in group size 30 with proportion (1: 1:1) (i.e., there were 10 low-confidence individuals, 10 medium-confidence individuals, and 10 high-confidence individuals in a group; called "diverse confidence"), along with results of groups in which only low-, medium-, or high-confidence individuals were present (i.e., identical to the results of group size 30 shown in Fig. 7). In both tasks, the mean accuracy rates were above the chance level (.50) in the diverse confidence groups. Furthermore, $95 \%$ confidence intervals of the accuracy were overlapped between confidence levels: Low [.69, .87], medium [.68, .86], high [.71, .89], and diverse [.70, .87] in a population inference task; low $[.38, .70]$, medium $[.39, .75]$, high $[.69, .96]$, and diverse $[.56, .83]$ in a relationships comparison task. These results suggest that even if low-confidence individuals are in a group, their judgments are unlikely to lead group judgments in inaccurate directions. That is, when making group judgments, accepting low-confidence individuals' judgments does not always decrease the accuracy of group judgments.

As described earlier, this study also simulated 10 proportion patterns in confidence levels (i.e., Low: Medium: High = 3: 1: 1, 2: 3: 0, and so on), for each group size (i.e., 5, 10, 20, and 30). However, these results showed similar tendencies for all proportions and group sizes. Therefore, we report only the results of group size 30 with 1:1:1 in this section, and show the other results in Supplementary Information 6.

\section{Discussion}

This study investigated the relationships between individuals' subjective confidence in their judgments and the accuracy of group judgments in binary choice tasks. According to the wisdom of crowds framework, aggregating individuals' judgments (e.g., by a majority rule) often leads to improve group judgment accuracy if the mean of rates of individuals' correct judgments is above the chance level and if they make diverse judgments between themselves. In this study, the analyses considered the following issues: Is it better to make groups with only high-confidence individuals? Would adding low-confidence individuals to a group lead to worse outcomes? Through behavioral experiments on individual judgments and computer simulations of group judgments, the present study revealed some interesting insights on these issues at individual and group levels. At individual levels, subjective confidence was not always highly correlated with accuracy of judgments among individuals. At group levels, the judgments of lowerconfidence individuals did not always impair the accuracy of group judgments, both in similar and diverse confidence groups. In sum, it is suggested that accepting low-confidence individuals' judgments does not always impair the accuracy of group judgments.

Based on the results of this study, two practical implications for group judgments are proposed. The first relates to the importance of accepting judgments not only by high-confidence individuals but also by lowconfidence individuals. The current findings showed that aggregating low-confidence individuals' 
judgments sometimes led to similar accuracy as aggregating high-confidence individuals' judgments (first computer simulation). In addition, even if there were low-confidence individuals in a group, the accuracy of group judgments was not always impaired (second computer simulation). Generally, it seems that individuals with high confidence have much knowledge about tasks (often experts) and are likely to make accurate group judgments. However, group judgments made in real-world contexts, it may be difficult to bring together many experts. Furthermore, some individuals may not have much knowledge and may have low confidence (often novices). Even if there are low-confidence individuals in a group, they may sometimes provide their judgments from a unique point of view that experts do not possess. It is implied that when many people discuss tasks and make group judgments, low-confidence individuals may sometimes bring new perspectives for solving tasks. At least, accepting low-confidence individuals' judgments will be unlikely to inhibit the accuracy of group judgments. The second implication for group judgments relates to the need to be careful to consider only superficial subjective confidence. Since individuals' confidence was not highly correlated with the accuracy of their judgments, adding lowerconfidence individuals into a group does not always inhibit group judgment accuracy; in other words, adding medium-confidence individuals instead of low-confidence individuals does not always improve the accuracy of group judgments. For example, the post hoc analyses of a relationships comparison task in group size 30 revealed that the accuracy of group judgments in proportion "2: 3: 0" (.56) did not improve than that in proportion " $3: 2: 0$ " (.56). As a recent study pointed out ${ }^{[27]}$, these findings imply that to achieve the wisdom of crowds, it is important to not only consider how confident individuals feel but also what they know (and do not know).

Finally, two limitations of this study regarding individuals' subjective confidence must be stated. The first limitation is the factors that may generate individuals' subjective confidence. Evidently, the amount of individuals' knowledge of tasks can be highly correlated with their confidence. If the familiarity with objects in a question (i.e., alternatives, or objects in a question sentence) was higher, then individuals' confidence in their judgments may also be higher, and vice versa. However, not only knowledge but also other factors, such as familiarity with objects, may also be related. It is speculated that confidence may be generated by the familiarity of only one object; rather, confidence may be strongly correlated with relative differences in familiarity between two or more objects. For example, the difference between the "difference in familiarity between city $Q$ and country A" and "difference in familiarity between city $Q$ and country B" (in a relationships comparison task) might be more critical for generating confidence than the familiarity of one city or one country itself. In fact, recent studies on perceptual decisions have shown that the differences in posterior probabilities (i.e., participants' subjective probability that the decision was correct) between the best and second-best alternatives could best explain their degree of confidence about the judgments ${ }^{[28],[29]}$. Future works need to further investigate the possible factors that are highly related to individuals' confidence. Clarifying such issues may enable the investigations of mechanisms that improve individuals' and groups' judgments in detail by modeling individuals' confidence. The second limitation is the method of aggregating individuals' judgments. Although only one majority rule was applied in this study, many previous studies on the wisdom of crowds have discussed different ways to effectively aggregate individuals' judgments for improving group judgments ${ }^{[6]}$, [30-32]. Some studies 
have argued the importance of considering subjective confidence in group judgments ${ }^{[30],[32}$. One of the typical aggregation methods using confidence is a method called a confidence-weighted majority rule, which is assumed that a group chooses one alternative whose sum of confidence ratings is higher. Consider the situation where 10 people's judgments are "AAAAAABBBB" in a binary choice task ( $A$ and $B$ denote two alternatives) and their confidence ratings are " $10,10,20,5,15,10,60,70,80,70$," respectively. In this situation, although a group judgment is $\mathrm{A}$ according to a simple majority rule, it is $\mathrm{B}$ according to a weighted-confidence majority rule because the sum of confidence ratings in $B(280)$ is larger than that in A (70). In addition, some previous studies have considered the confidence threshold of accepting

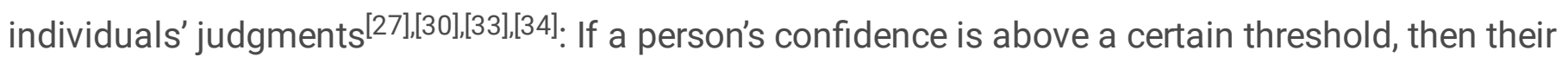
judgment is accepted, and vice versa. This procedure is repeated until all the group members' judgments are evaluated. Previous studies have investigated group sizes and the optimal confidence threshold for achieving the wisdom of crowds, using computer simulations. Based on such studies, it is necessary to also investigate group judgments' performances by applying previously proposed methods and to confirm the generalizability of the results of this study.

\section{Conclusions}

According to the framework of the wisdom of crowds, the present study investigated whether lowconfidence individuals would inhibit group judgments' accuracy in a binary choice task. Through behavioral experiments on individual judgments and computer simulations of group judgments, it was found that low-confidence individuals could sometimes make accurate group judgments similar to highconfidence individuals, as the group size increased. It was also found that group judgments were unlikely to be biased (i.e., the mean of accuracy fell below the chance level, .50) even if there were low-confidence individuals in a group. However, it was implied that because individuals' confidence was not highly correlated with their accuracy of judgments, adding higher-confidence individuals (e.g., medium confidence) into a group did not always contribute to improve the accuracy of group judgments. This study not only will expand understanding on the wisdom of crowds framework in terms of subjective confidence but also may provide practical implications for group judgments (e.g., whose judgment people should accept). In conclusion, taking low-confidence individuals' judgments into account does not always decrease the accuracy of group judgments.

\section{Declarations}

\section{Author contributions}

M.S. and H.H. designed the study. M.S. gathered and analyzed the data. M.S. and H.H. wrote the manuscript.

\section{Competing interests}


The authors declare no competing interests.

\section{Data availability}

All data, code, and materials used in this study are available at

https://osf.io/ehzu6/?view_only=fb08ab0fc87c45eabb201316bd2c0881.

\section{References}

1. Galton, F. Vox populi. Nature, 75, 450-451 (1907).

2. Surowiecki, J. The wisdom of crowds. Anchor, https://doi.org/10.1016/S0140-6736(16)31130-8 (2004).

3. Lorenz, J., Rauhut, H., Schweitzer, F. \& Helbing, D. How social influence can undermine the wisdom of crowd effect. Proc. Natl. Acad. Sci. U. S. A. 108, 9020-9025(2011).

4. Keck, S. \& Tang, W. Enhancing the Wisdom of the Crowd With Cognitive Process Diversity: The Benefits of Aggregating Intuitive and Analytical Judgments. Psychol. Sci, 1-11 https://doi.org/10.2139/ssrn.3319676 (2020).

5. Navajas, J., Niella, T., Garbulsky, G., Bahrami, B. \& Sigman, M. Aggregated knowledge from a small number of debates outperforms the wisdom of large crowds. Nat. Hum. Behav, 2, 126-132 (2018).

6. Palley, A. B. \& Soll, J. B. Extracting the wisdom of crowds when information is shared. Manage. Sci, 65, 2291-2309 (2019).

7. Herzog, S. M. \& Hertwig, R. Harnessing the wisdom of the inner crowd. Trends Cogn. Sci, 18, 504-506 (2014).

8. Vul, E. \& Pashler, H. Measuring the Crowd Within: Probabilistic Representation Within Individuals. Psychol. Sci, 19, 645-647 (2008).

9. Palley, A. B. Extending the Wisdom of Crowds: Quantifying Uncertainty Using the Mean and Variance of a Collection of Point Estimates. SSRN Electron. J, 1-19 https://doi.org/10.2139/ssrn.3014058 (2017).

10. Herzog, S. M., Litvinova, A., Yahosseini, K. S., Tump, A. N. \& Kurvers, R. H. J. M. The Ecological Rationality of the Wisdom of Crowds. in Taming Uncertainty245-262(The MIT Press, 2019).

11. Thomas, B., Coon, J., Westfall, H. A. \& Lee, M. D. Model-based Wisdom of the Crowd for Sequential Decision-Making Tasks. Cogn. Sci, 45, e13011 (2021).

12. Honda, H., Abe, K., Matsuka, T. \& Yamagishi, K. The role of familiarity in binary choice inferences. Mem. Cognit, 39, 851-863 (2011).

13. Honda, H., Matsuka, T. \& Ueda, K. Memory-Based Simple Heuristics as Attribute Substitution: Competitive Tests of Binary Choice Inference Models. Cogn. Sci, 41, 1093-1118 (2017). 
14. Goldstein, D. G. \& Gigerenzer, G. Models of Ecological Rationality: The Recognition Heuristic. Psychol. Rev, 109, 75-90 (2002).

15. Marewski, J. N. \& Mehlhorn, K. Using the ACT-R architecture to specify 39 quantitative process models of decision making. Judgm. Decis. Mak, 6, 439-519 (2011).

16. Gigerenzer, G. \& Goldstein, D. G. Reasoning the Fast and Frugal Way: Models of Bounded Rationality. Psychol. Rev, 103, 650-669 (1996).

17. Shirasuna, M., Honda, H., Matsuka, T. \& Ueda, K. Familiarity-matching in decision making: Experimental studies on cognitive processes and analyses of its ecological rationality. in Proceedings of the 39th Annual Conference of the Cognitive Science Society (eds. Gunzelmann G., Howes A., Tenbrink T. \& Davelaar E. J.) 3143-3148 (Cognitive Science Society, 2017).

18. Shirasuna, M., Honda, H., Matsuka, T. \& Ueda, K. Familiarity-Matching: An Ecologically Rational Heuristic for the Relationships-Comparison Task. Cogn. Sci, 44, e12806 (2020).

19. Shirasuna, M. The adaptive use of heuristics: Investigations of human inferential strategies in a new task structure. (Ph.D Thesis at The University of Tokyo, 2021).

20. Erdfelder, E., FAul, F., Buchner, A. \& Lang, A. G. Statistical power analyses using G*Power 3.1: Tests for correlation and regression analyses. Behav. Res. Methods, 41, 1149-1160 (2009).

21. Hoffrage, U. \& Reimer, T. Models of Bounded Rationality: The Approach of Fast and Frugal Heuristics. Manag. Revu, 15, 437-459 (2004).

22. Gigerenzer, G. \& Gaissmaier, W. Heuristic Decision Making. Annu. Rev. Psychol, 62, 451-482 (2011).

23. Gigerenzer, G. \& Goldstein, D. G. The recognition heuristic: A decade of research. Judgm. Decis. Mak, 6, 100-121 (2011).

24. Coutinho, M. V. C., Thomas, J., Alsuwaidi, A. S. M. \& Couchman, J. J. Dunning-Kruger Effect: Intuitive Errors Predict Overconfidence on the Cognitive Reflection Test. Front. Psychol, 1-10 https://doi.org/10.3389/fpsyg.2021.603225 (2021).

25. Moore, D. A. \& Healy, P. J. The Trouble With Overconfidence. Psychol. Rev, 115, 502-517 (2008).

26. Kruger, J. \& Dunning, D. Unskilled and unaware of it: how difficulties in recognizing one's own incompetence lead to inflated self-assessments. J. Pers. Soc. Psychol, 77, 1121-1134 (1999).

27. Silver, I., Mellers, B. A. \& Tetlock, P. E. Wise teamwork: Collective confidence calibration predicts the effectiveness of group discussion. J. Exp. Soc. Psychol, 96, 104157 (2021).

28. Rahnev, D. Confidence in the Real World. Trends Cogn. Sci, 24, 590-591 (2020).

29. Li, H. H. \& Ma, W. J. Confidence reports in decision-making with multiple alternatives violate the Bayesian confidence hypothesis. Nat. Commun, 11, 1-11 (2020).

30. Moussaïd, M. \& Yahosseini, K. S. Can Simple Transmission Chains Foster Collective Intelligence in Binary-Choice Tasks? PLoS One, 11, e0167223 (2016).

31. Hamada, D., Nakayama, M. \& Saiki, J. Wisdom of crowds and collective decision-making in a survival situation with complex information integration. Cogn. Res. Princ. Implic, 5, 1-15 (2020). 
32. Meyen, S., Sigg, D. M. B., von Luxburg, U. \& Franz, V. H. Group decisions based on confidence weighted majority voting. Cogn. Res. Princ. Implic, 6, 1-13 (2021).

33. Pescetelli, N., Haupericha, A. K. \& Yeunga, N. Confidence, advice seeking and changes of mind in decision making., 215, 1-9 (2021).

34. Soll, J. B., Palley, A. \& Rader, C. The Bad Thing About Good Advice: Understanding When and How Advice Exacerbates Overconfidence. Manage. Sci, https://doi.org/10.2139/ssrn.3094176 (2020).

\section{Figures}

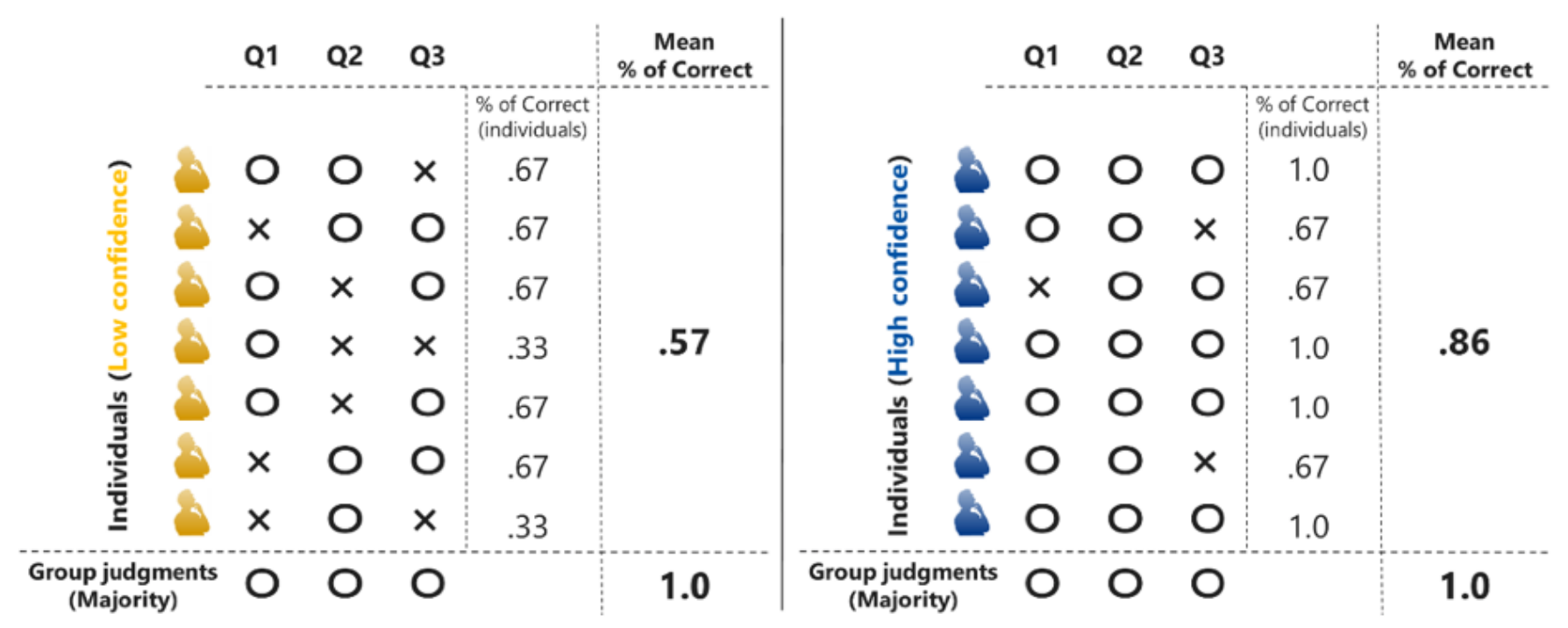

\section{Figure 1}

Hypothetical examples of the role of diversity of judgments in a majority rule (three questions, seven individuals) in binary choice tasks. Circles and crosses denote correct and false judgments, respectively. In individual levels, low-confidence individuals may make less accurate judgments than high-confidence individuals (the means of rates of correct judgments are .57 and .86 in low- and high-confidence individuals, respectively) because, for example, low-confidence individuals make judgments based on their lesser knowledge that involves uncertainty. In group levels, however, a low-confidence group can sometimes make judgments as accurate as a high-confidence group by using a majority rule (the rates of correct judgments are .75 in both groups) because of diversity of judgments by low-confidence individuals. 


\section{Population inference task}

\section{人口の多い都市はどちらだと思いますか（Which city has a larger population?）}

\section{○都城市}

\section{(Miyakonojo-shi)}

日立市

(Hitachi-shi)

今の回答に、どのくらい自信がありましたか

(How confident do you feel for your judgment?)

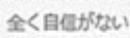
(not confident at all)
(highly confident)

\section{Relationships comparison task}

キエフという都市がある国はどちらだと思いますか

(Which country is Kiev in?)

○ワクライナ

(Ukraine)

○ イラク

(Iraq)

今の回答に、どのくらい自信がありましたか（How confident do you feel for your judgment?）

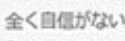

(not confident at all) ${ }^{50} \quad$ (highly confident)

Figure 2

Screenshots of experimental tasks. Descriptions in parentheses are the translated descriptions from Japanese. Upper panel: Population inference task. Lower panel: Relationships comparison task. 


\section{Computer simulation (Similar confidence group)}

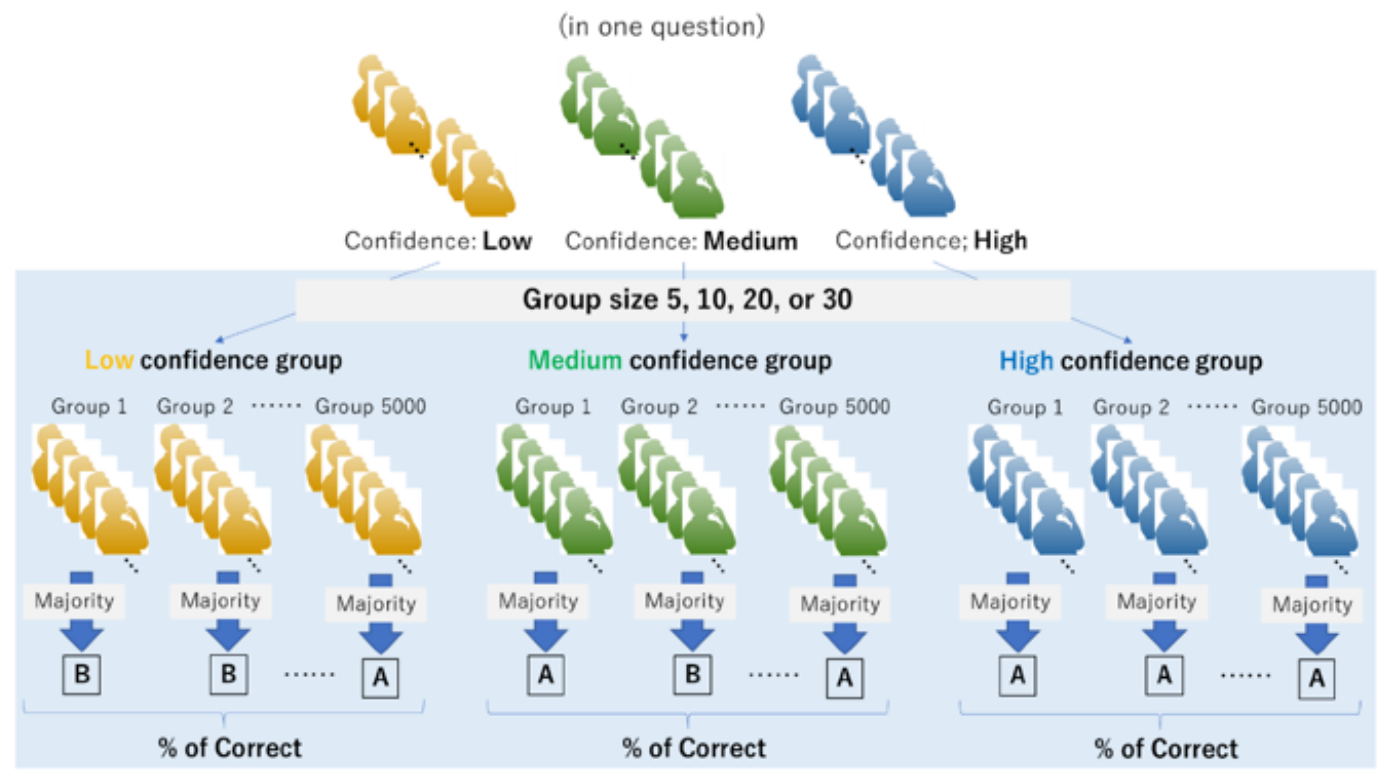

\section{Computer simulation (Diverse confidence group)}

(in one question)
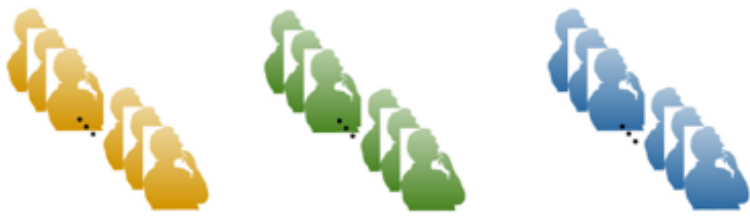

Confidence: Low Confidence: Medium Confidence; High

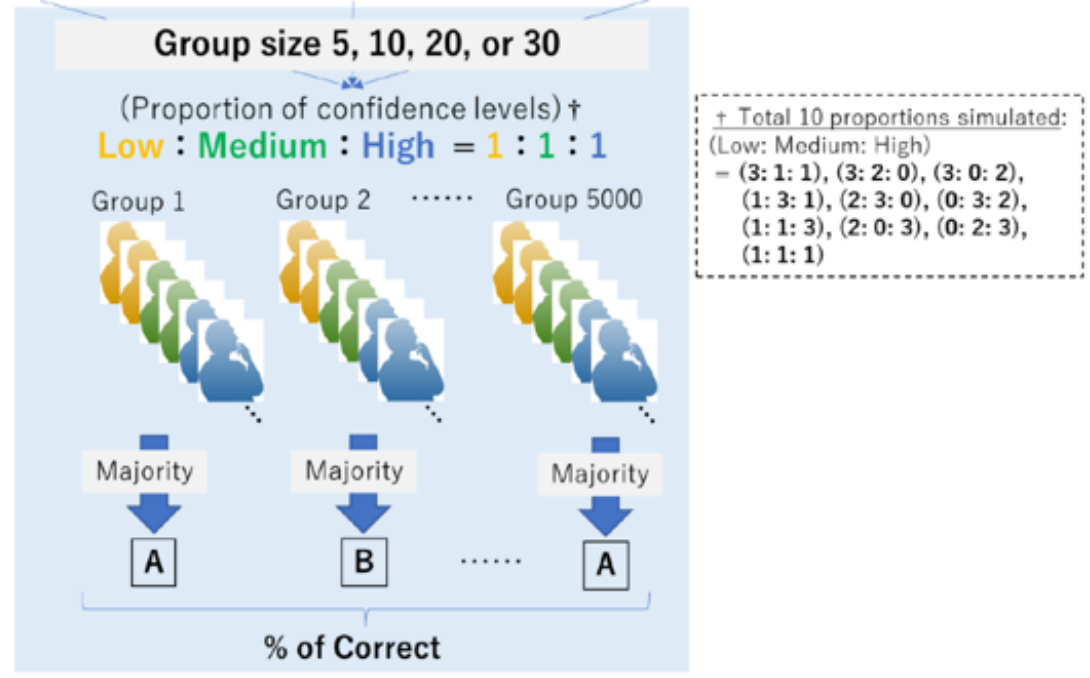

\section{Figure 3}

Schematic of computer simulations in the development of group judgments. Upper: Similar confidence groups. Lower: Diverse confidence groups. 
Relationships between Confidence and Accuracy

(Examples in Population Inference Task; set A)

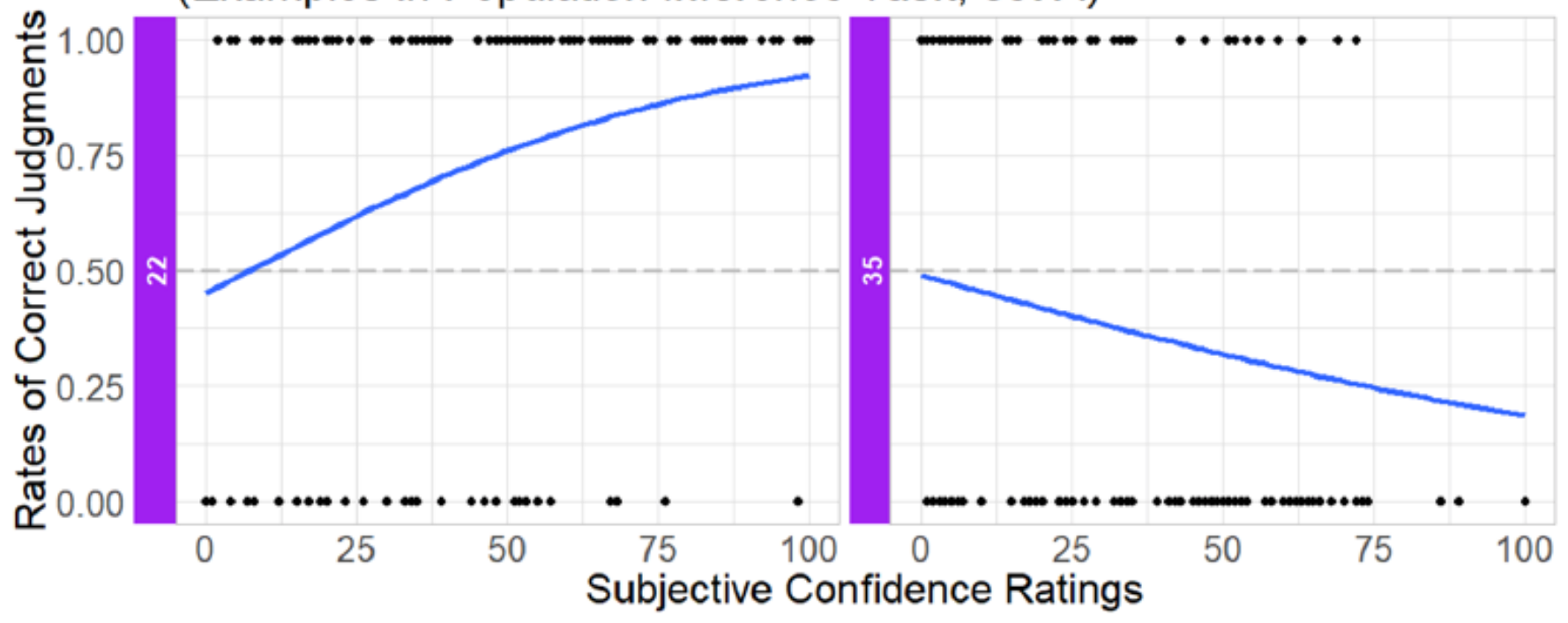

Relationships between Confidence and Accuracy (Examples in Relationships Comparison Task; set A)

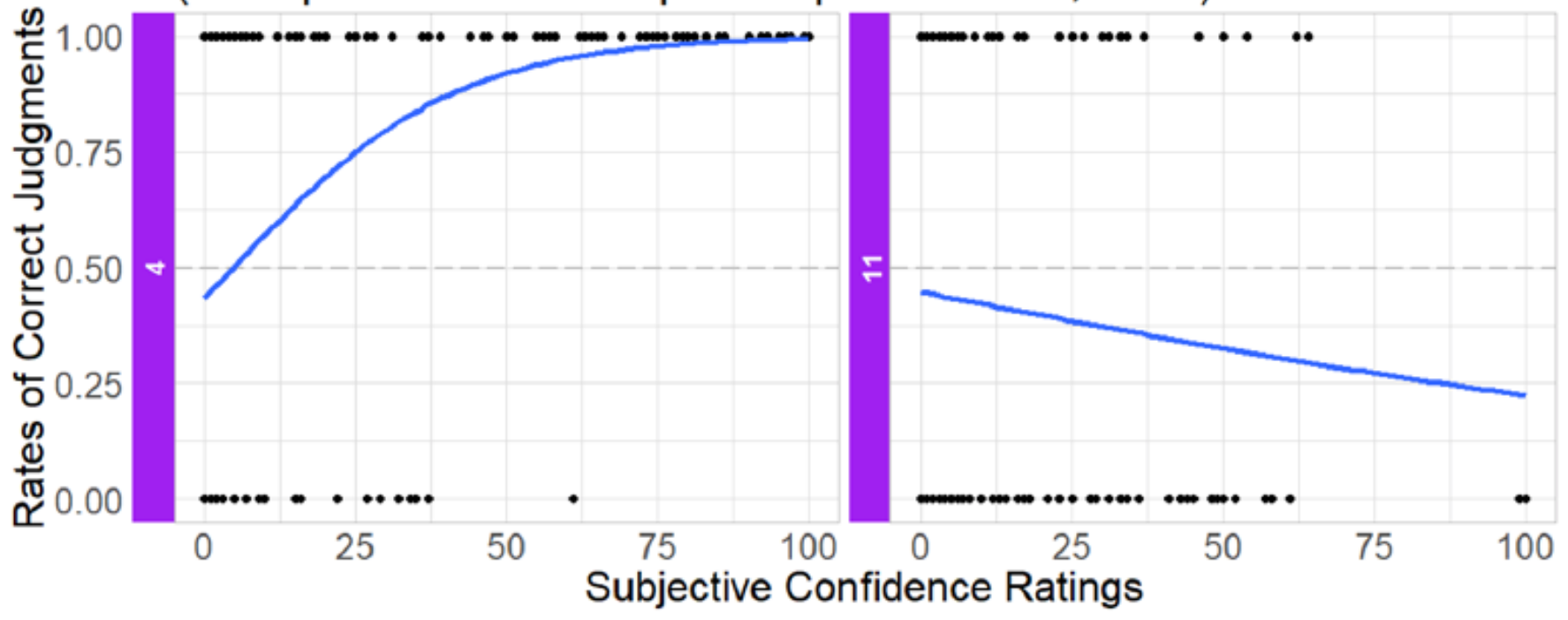

Figure 4

Examples of relationships between individuals' confidence (subjective confidence ratings; $x$-axis) and their accuracy (rates of correct judgments; $y$-axis) in behavioral experiments. Each point denotes individuals' choices: Correct (1) or false (0). Blue lines denote regression curves by generalized linear models. Dotted gray lines indicate the chance level in a binary choice task (i.e., .50). Numbers in purple squares show the question numbers in the experimental tasks. Left columns show examples of positive relationships between confidence and accuracy, while right columns show those of negative relationships. Upper panel: Population inference task. Lower panel: Relationships comparison task. 
Distribution of Coefficients of Confidence in Generalized Linear Model (Population Inference Task; set A)

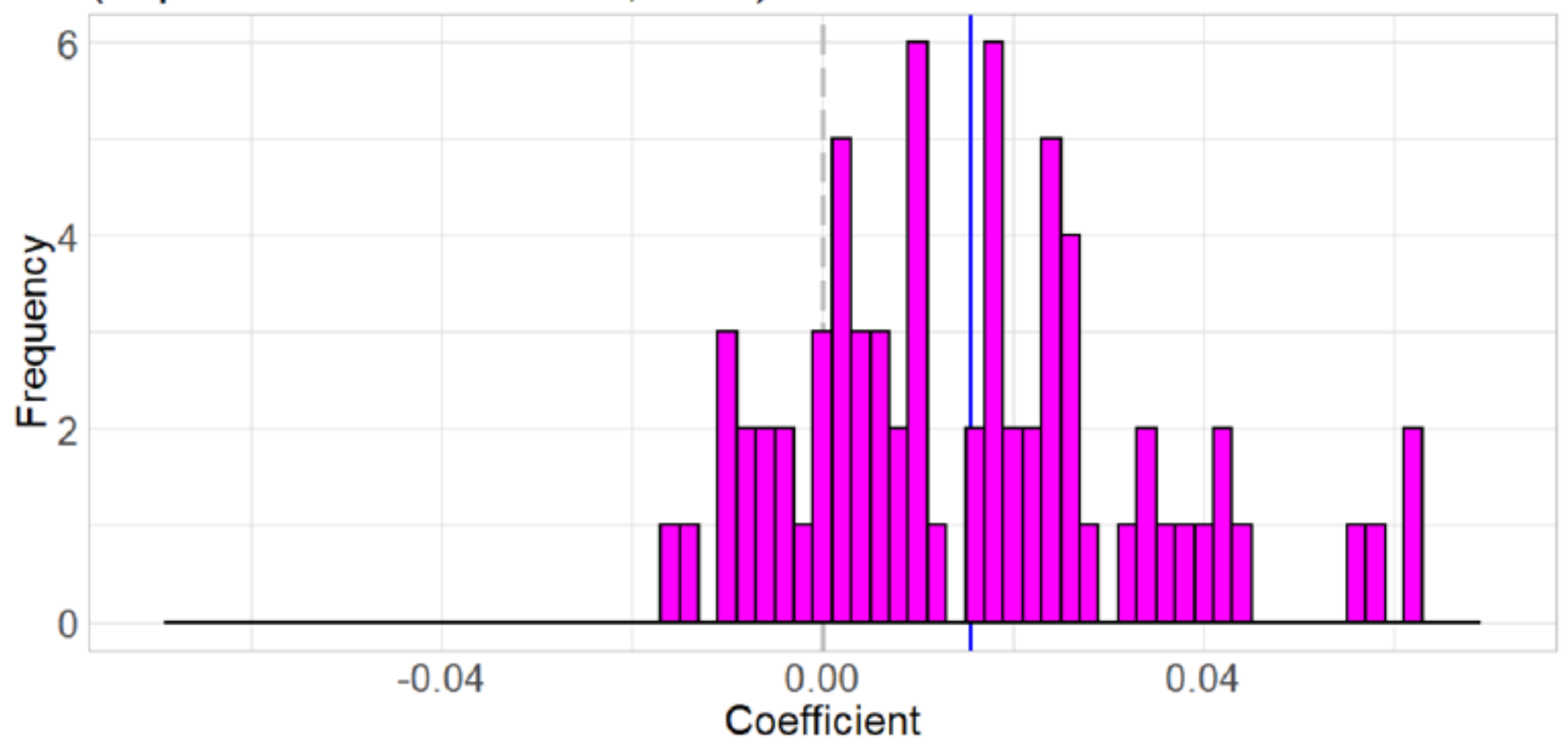

Distribution of Coefficients of Confidence in Generalized Linear Model (Relationships Comparison Task; set A)

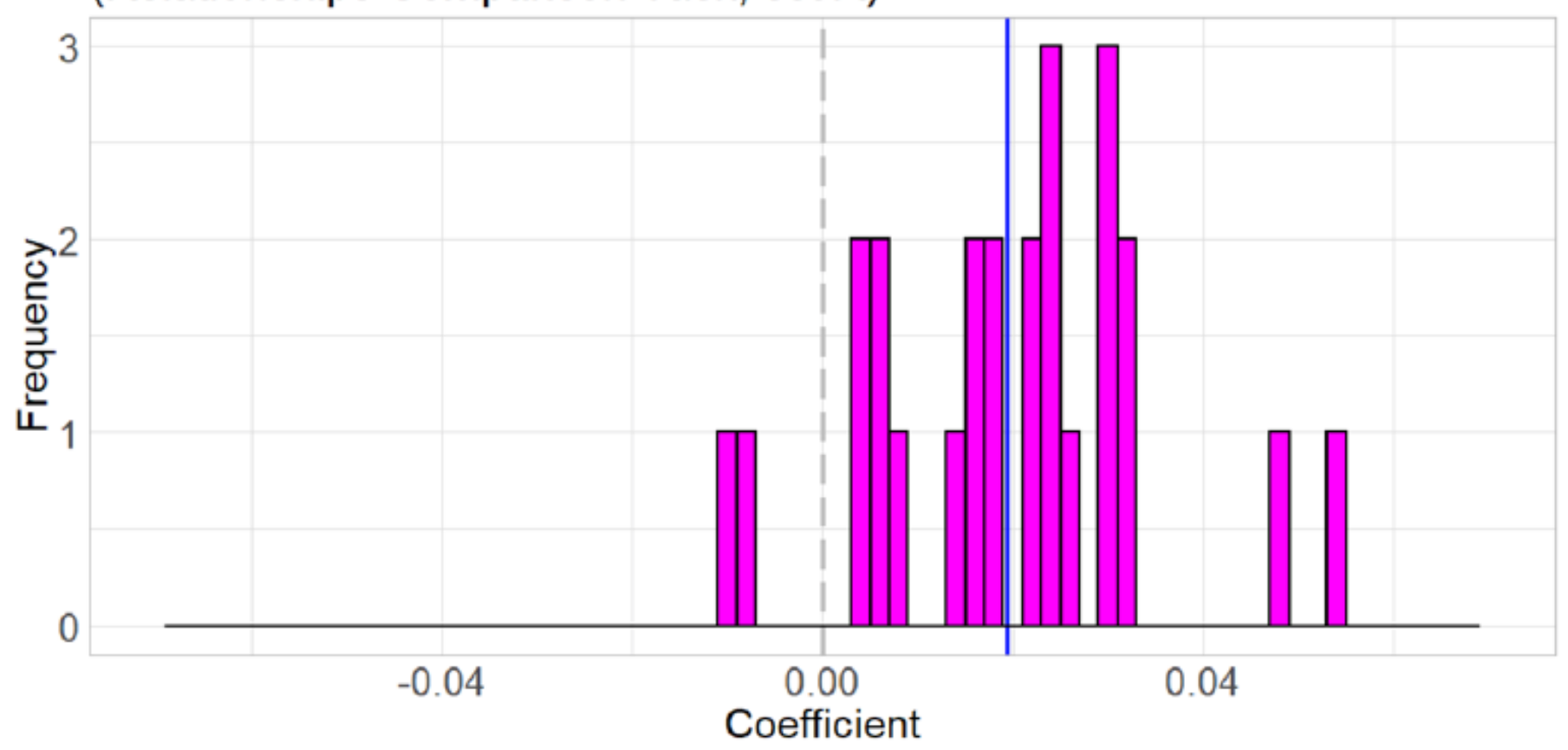

Figure 5

Distributions of coefficients of confidence in the generalized linear models. Blue lines denote the means of coefficients ( 0.015 and 0.019 , in a population inference task and in a relationships comparison task, respectively). Upper panel: Population inference task. Lower panel: Relationships comparison task. 
Accuracy (Population Inference Task; Set A)
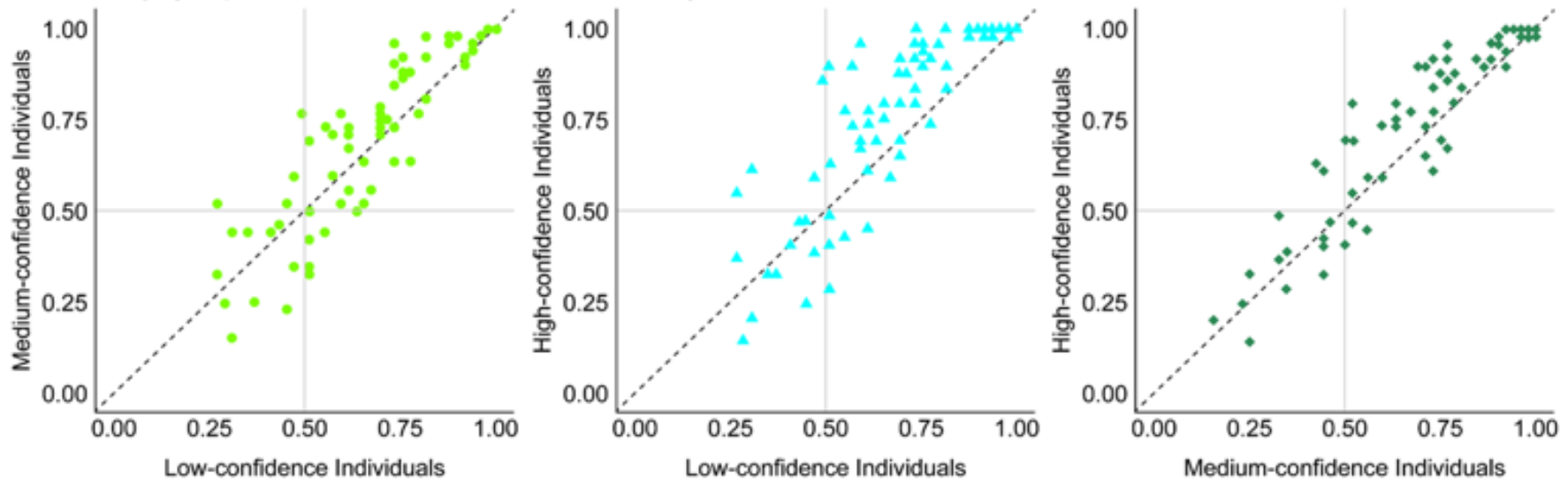

\section{Accuracy (Relationships Comparison Task; Set A)}
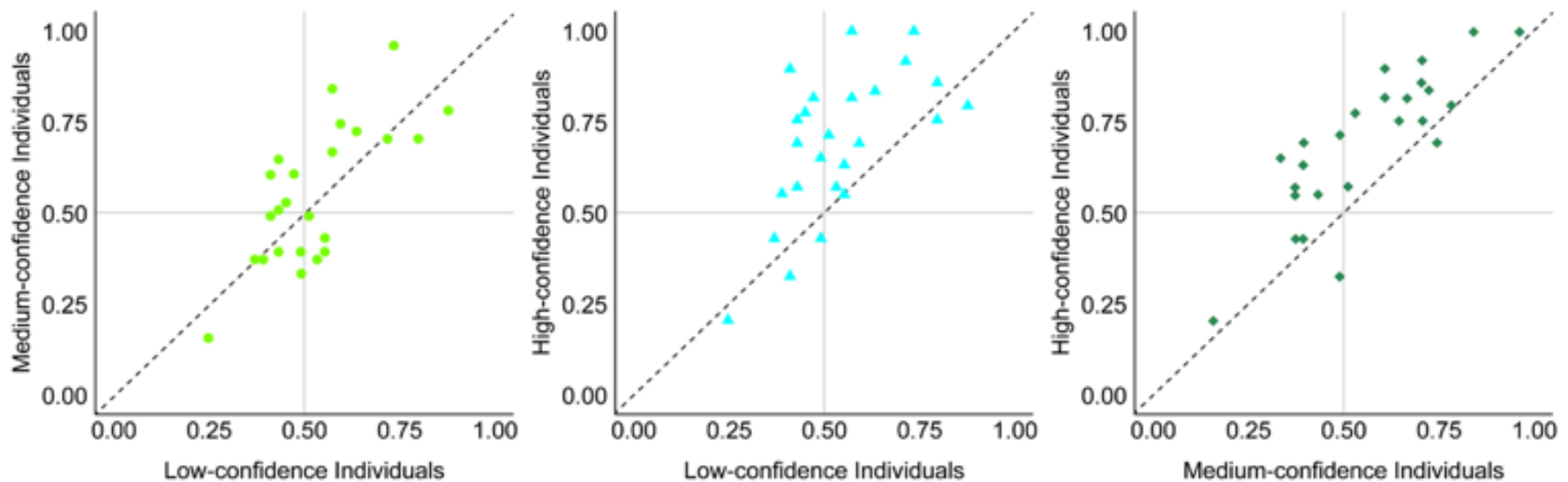

\section{Figure 6}

Proportions of correct judgments for each confidence level in individual levels. Each point denotes the rate of correct judgments for each question. Upper panel: Population inference task (70 questions). Lower panel: Relationships comparison task (25 questions). Left, middle, and right graphs show the relationships between low- and medium-confidence individuals' accuracy, relationships between low- and high-confidence individuals' accuracy, and relationships between medium- and high-confidence individuals' accuracy, respectively. Note: Spearman rank sum correlations rho were as follows. Lowmedium .90, Low-high .88, Medium-high .94 in a population inference task; Low-medium .72, Low-high .60 , Medium-high .81 in a relationships comparison task; all ps $<.001$ 


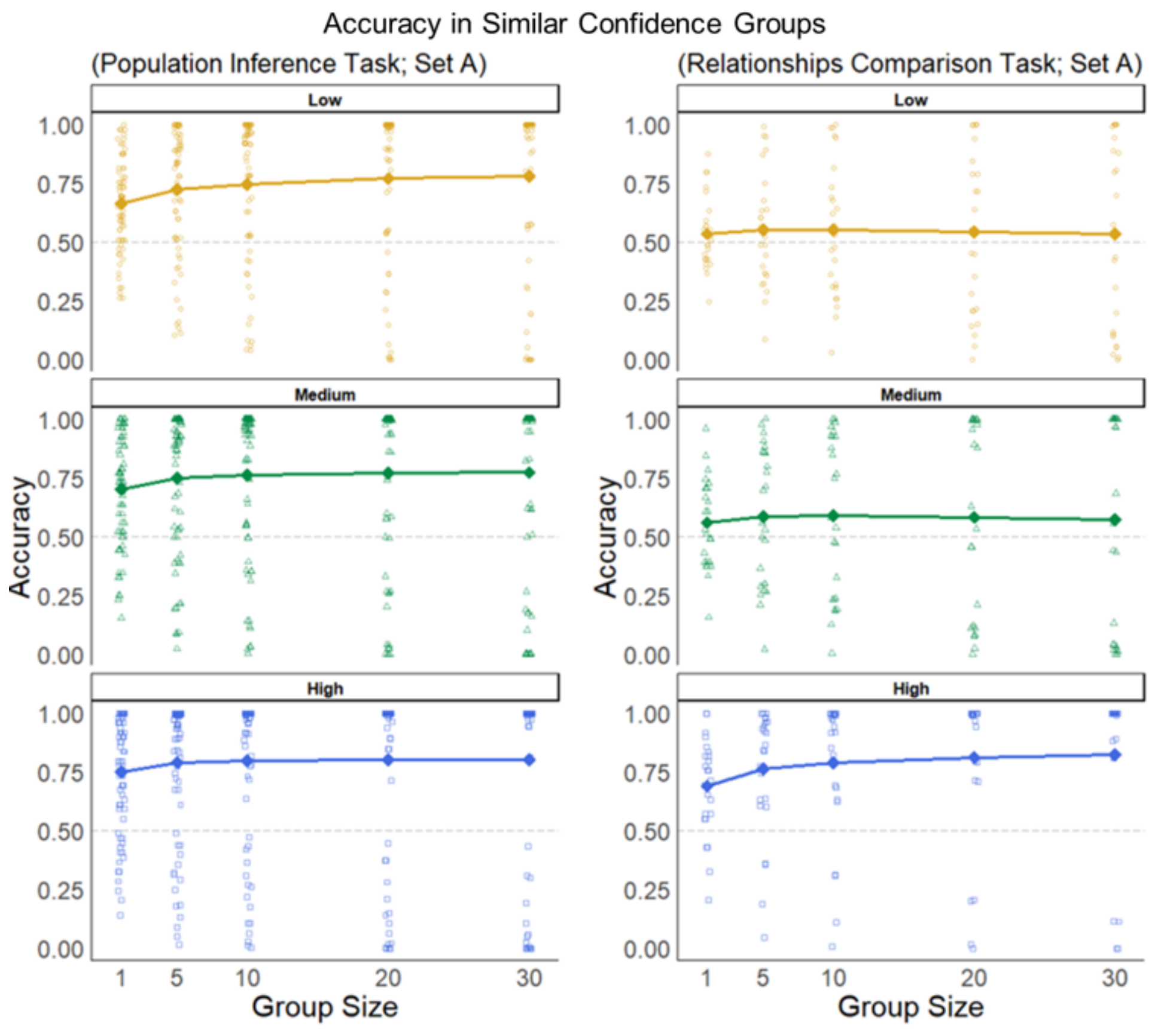

Figure 7

Results of computer simulations for similar confidence groups. X-axis denotes group sizes 1 (individual), $5,10,20$, and 30; and y-axis denotes group judgments' accuracy (i.e., rates of correct judgments). Dotted lines and each point indicate the chance level of accuracy (.50) and accuracy for each question, respectively. Lines connect the means of accuracy in each group size. Top, middle, and bottom panels denote the groups comprising low-, medium-, and high-confidence individuals, respectively. Upper: Population inference task. Lower: Relationships comparison task. 
Accuracy in Similar and Diverse Confidence Groups (Group Size 30) (Population Inference Task; Set A)
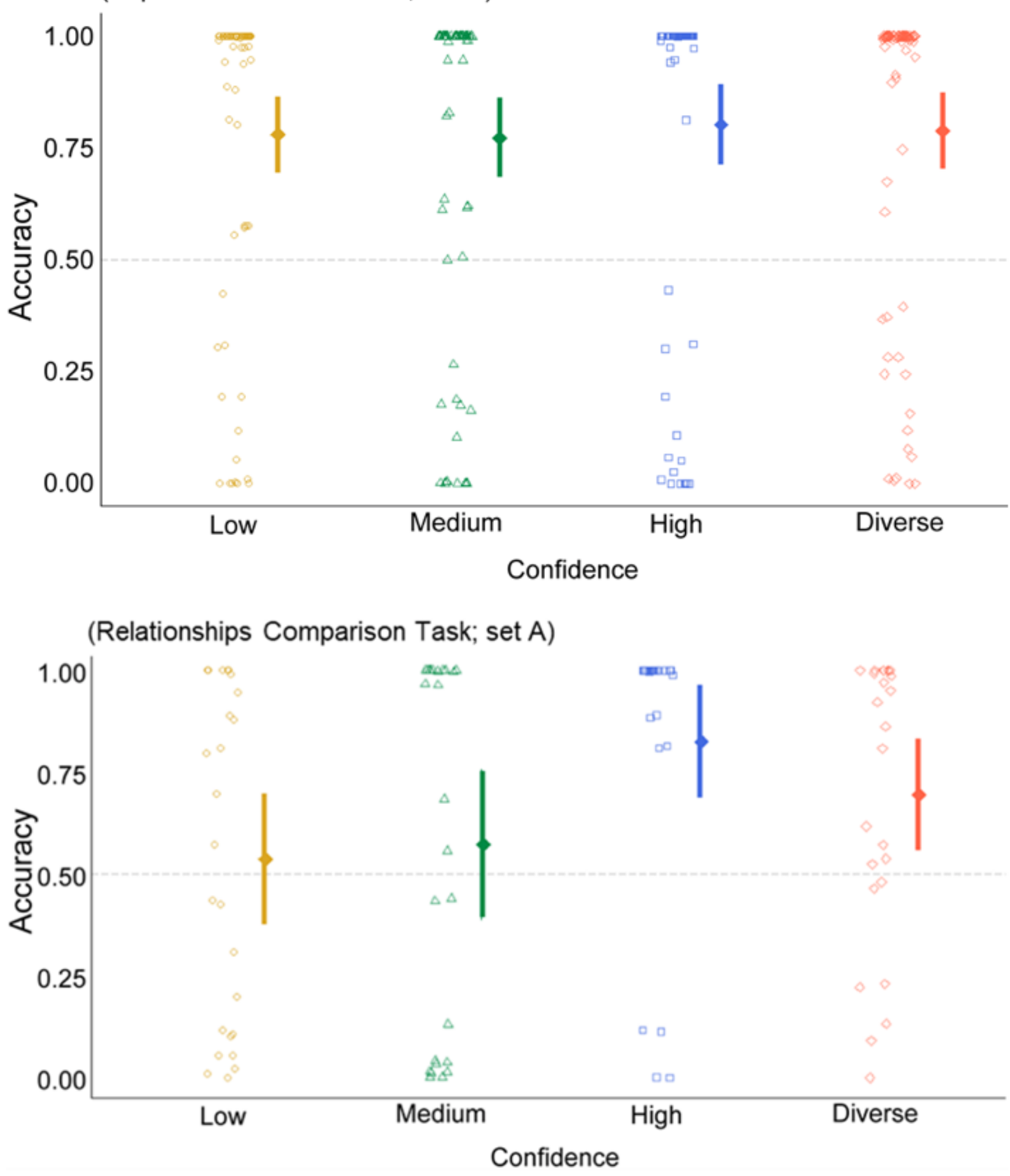

Figure 8

Results of computer simulations for diverse confidence groups in group size 30. The term "Diverse" denotes groups in which there were 10 low-confidence, 10 medium-confidence, and 10 high-confidence individuals. Results in "Low," "Medium," and "High" are identical to Fig. 7 in group size 30. Dotted lines and each point indicate the chance level of accuracy (.50) and the accuracy for each question, respectively. Error bars and bigger points show 95\% confidence intervals the mean of accuracy (low .78, 
medium .77, high .80, diverse .79 in a population inference task; low .52, medium .57, high .82, diverse .69 in a relationships comparison task). Upper: Population inference task. Lower: Relationships comparison task.

\section{Supplementary Files}

This is a list of supplementary files associated with this preprint. Click to download.

- submv1210826v2supScirep.pdf 